\title{
Optimal double-loop networks with non-unit steps*
}

\author{
F. Aguiló, E. Simó and M. Zaragozá \\ Dept. de Matemàtica Aplicada IV \\ Universitat Politècnica de Catalunya \\ C/. Jordi Girona 1-3 \\ 08034 Barcelona, Spain. \\ matfag@mat.upc.es
}

Submitted: Apr 1, 2002; Accepted: Dec 19, 2002; Published: Jan 6, 2003

MR Subject Classifications: 05C20, 05C12, 05C85, 68M10.

\begin{abstract}
A double-loop digraph $G\left(N ; s_{1}, s_{2}\right)=G(V, E)$ is defined by $V=\mathbb{Z}_{N}$ and $E=\left\{\left(i, i+s_{1}\right),\left(i, i+s_{2}\right) \mid i \in V\right\}$, for some fixed steps $1 \leq s_{1}<s_{2}<N$ with $\operatorname{gcd}\left(N, s_{1}, s_{2}\right)=1$. Let $D\left(N ; s_{1}, s_{2}\right)$ be the diameter of $G$ and let us define

$$
D(N)=\min _{\substack{1 \leq s_{1}<s_{2}<N, \operatorname{gcd}\left(N, s_{1}, s_{2}\right)=1}} D\left(N ; s_{1}, s_{2}\right), \quad D_{1}(N)=\min _{1<s<N} D(N ; 1, s) .
$$

Some early works about the diameter of these digraphs studied the minimization of $D(N ; 1, s)$, for a fixed value $N$, with $1<s<N$. Although the identity $D(N)=$ $D_{1}(N)$ holds for infinite values of $N$, there are also another infinite set of integers with $D(N)<D_{1}(N)$. These other integral values of $N$ are called non-unit step integers or nus integers.

In this work we give a characterization of nus integers and a method for finding infinite families of nus integers is developed. Also the tight nus integers are classified. As a consequence of these results, some errata and some flaws in the bibliography are corrected.
\end{abstract}

Keywords: Diameter, double-loop network, nus integer, optimal family, L-shaped tile, Smith normal form.

\footnotetext{
${ }^{*}$ Work supported by the Ministry of Science and Technology, Spain, and the European Regional Development Fund (ERDF) under project TIC-2001 2171 and by the Catalan Research Council under project 2000SGR00079.
} 


\section{Notation and preliminary results}

Double-loop digraphs $G=G\left(N ; s_{1}, s_{2}\right)$, with $1 \leq s_{1}<s_{2}<N$ and $\operatorname{gcd}\left(N, s_{1}, s_{2}\right)=1$, have the vertex set $V=\mathbb{Z}_{N}$ and the adjacencies are defined by $v \rightarrow v+s_{i}(\bmod N)$ for $v \in V$ and $i=1,2$. The hops $s_{1}$ and $s_{2}$ between vertices are called steps.

These kind of digraphs have been widely studied to modelize some local area networks, known as double-loop networks (DLN.) From the metric point of view, the minimization of the diameter of $G$ corresponds to a faster transmission of messages in the network.

The diameter of $G$ is denoted by $D\left(N ; s_{1}, s_{2}\right)$. As $G$ is vertex symmetric, its diameter can be computed from the expression $\max _{i \in V} d(0, i)$, where $d(u, v)$ is the distance from $u$ to $v$ in $G$. For a fixed $N \in \mathbb{N}$, the optimal value of the diameter is denoted by

$$
D(N)=\min _{\substack{1 \leq s_{1}<s_{2}<N, \operatorname{gcd}\left(N, s_{1}, s_{2}\right)=1}} D\left(N ; s_{1}, s_{2}\right) .
$$

Several works studied the minimization of the diameter (for a fixed $N$ ) with $s_{1}=1$. Let us denote $D_{1}(N)=\min _{1<s<N} D(N ; 1, s)$. Since the work of Wong and Coppersmith [7], a sharp lower bound is known for $D_{1}(N)$ :

$$
D_{1}(N) \geq\lceil\sqrt{3 N}\rceil-2=\operatorname{lb}(N) .
$$

Fiol et al. in [5] showed that $\operatorname{lb}(N)$ is also a sharp lower bound for $D(N)$. A given double-loop $G\left(N ; s_{1}, s_{2}\right)$ is called $k$-tight if $D\left(N ; s_{1}, s_{2}\right)=\operatorname{lb}(N)+k$. A $k$-tight DLN is called optimal if $D(N)=\mathrm{lb}(N)+k$. The 0-tight DLN are known as tight ones and they are also optimal. A full classification of tight and $k$-tight DLN can be found in [4] and [1], respectively.

The metrical properties of $G\left(N ; s_{1}, s_{2}\right)$ are fully contained in its related L-shaped tile $L(l, h, w, y)$ with area $N=l h-w y$. This tile can be obtained from $G$ with the following procedure:

1. In the squared plane, label each square with a number in $\{0,1,2, \ldots, N-1\}$ using the rules in the left side of Figure 1. All the additions must be taken modulus $N$.

2. Take any square labelled with ' 0 '. Associate to this square the squares with labels in $\{1,2, \ldots, N-1\}$ at minimum distance from ' 0 ' in the digraph $G$. Take a lexicographic order if necessary. The right side of Figure 1 shows the linked tile (and the tessellation it defines) to the digraph $G(7 ; 2,3)$. The linked tile of $G(7 ; 2,3)$ has dimensions $l=h=3, w=1$ and $y=2$.

It is always possible to form an L-shaped tile proceeding in this way [5, 7]. It is said that the tile $L$ can be $\left(s_{1}, s_{2}\right)$-implemented. Note that Chen and Hwang in [3] proposed the following necessary and sufficient conditions for a tile with area $N$ :

Theorem 1 There exists $G\left(N ; s_{1}, s_{2}\right)$ realizing the L-shape $(l, h, w, y)$ iff $l>y, h \geq w$ and $\operatorname{gcd}(l, h, w, y)=1$. 


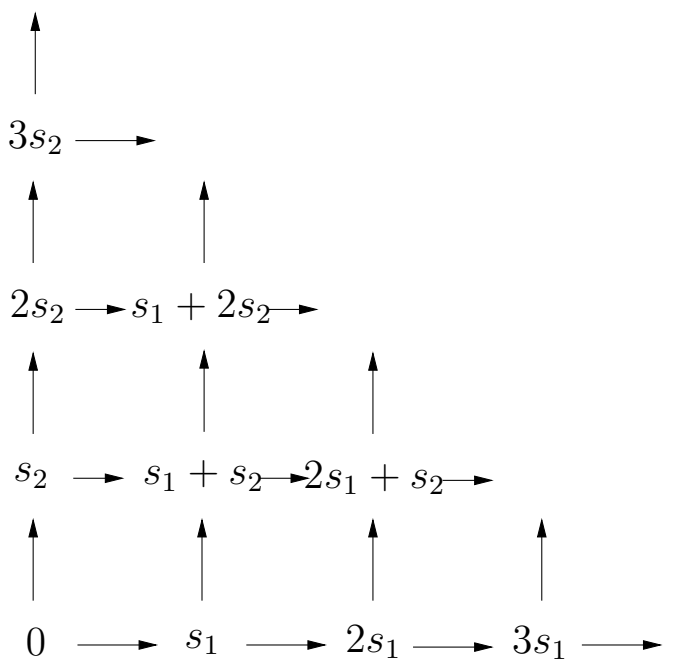

\begin{tabular}{|c|c|c|c|c|c|c|c|}
\hline 6 & 1 & 3 & 5 & 0 & 2 & 4 & 6 \\
\hdashline 3 & 5 & 0 & 2 & 4 & 6 & 1 & 3 \\
\hdashline 0 & 2 & 4 & 6 & 1 & 3 & 5 & 0 \\
\hline 4 & 6 & 1 & 3 & 5 & 0 & 2 & 4 \\
\hline 1 & 3 & 5 & 0 & 2 & 4 & 6 & 1 \\
\hdashline 5 & 0 & 2 & 4 & 6 & 1 & 3 & 5 \\
\hline 2 & 4 & 6 & 1 & 3 & 5 & 0 & 2 \\
\hline
\end{tabular}

Figure 1: Interconnection rules from a generic digraph $G\left(N ; s_{1}, s_{2}\right)$ and the related tile to $G(7 ; 2,3)$
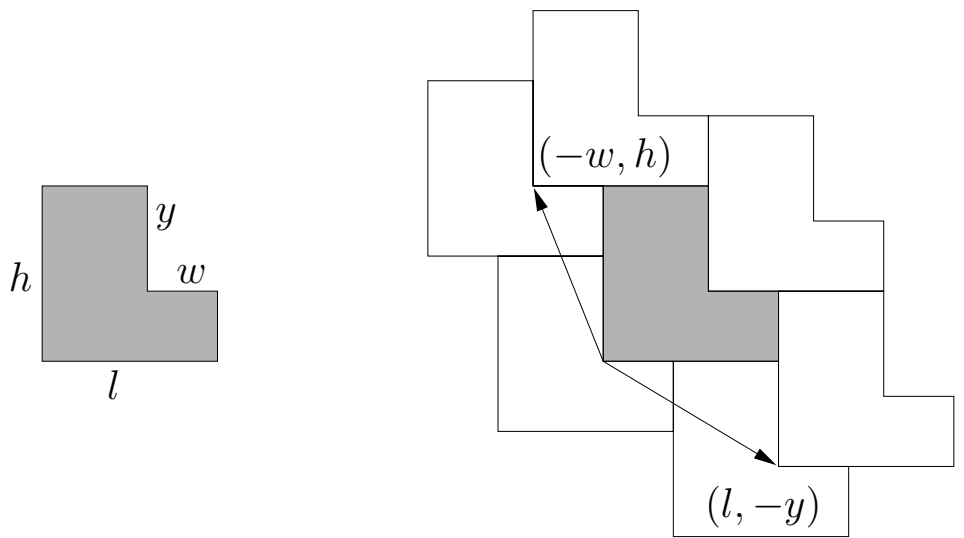

Figure 2: Generic dimensions of an L-shaped tile and its related tessellation.

Figure 2 describes how we denote the dimensions of a generic L-shaped tile and how the resulting tiling of the plane can be fully described from the integral matrix $M=$ $\left(\begin{array}{rr}l & -w \\ -y & h\end{array}\right)$, whose entries are the (column) vectors $\boldsymbol{u}=(l,-y)^{\top}$ and $\boldsymbol{v}=(-w, h)^{\top}$. In particular, the diameter's computation of $G$ can be done from the dimensions $L(l, h, w, y)$ by

$$
d(L)=d(L(l, h, w, y))=\max \{l+h-w-2, l+h-y-2\} .
$$

For obvious reasons, the value $d(L)$ is called the diameter of the tile $L$. It can be shown that $D\left(N ; s_{1}, s_{2}\right) \leq d(L)$ if $L$ is any linked tile to $G\left(N ; s_{1}, s_{2}\right)$. Several tiles can be related to a given digraph $G$ (possibly with different diameters,) however the tile generated by the previous procedure has the same diameter as $G$. In particular, when $d(L)=\operatorname{lb}(N)$ we have $d(L)=D\left(N ; s_{1}, s_{2}\right)=D(N)$. 
Definition 1 (Isomorphism of digraphs) Two digraphs, $G_{1}\left(V_{1}, E_{1}\right)$ and $G_{2}\left(V_{2}, E_{2}\right)$, are isomorphic if there is a bijective map $\phi: V_{1} \rightarrow V_{2}$ which preserves adjacencies, that is $(u, v) \in E_{1}$ iff $(\phi(u), \phi(v)) \in E_{2}$. Two isomorphic digraphs will be denoted by $G_{1} \cong G_{2}$.

As an immediate example of double-loop digraph isomorphism, we have $G\left(N ; s_{1}, s_{2}\right) \cong$ $G\left(N ; s_{2}, s_{1}\right)$ by the group isomorphism $\phi: \mathbb{Z}_{N} \rightarrow \mathbb{Z}_{N}$ given by $\phi\left(s_{1}\right)=s_{2}$ and $\phi\left(s_{2}\right)=s_{1}$ (provided that $\operatorname{gcd}\left(N, s_{1}, s_{2}\right)=1$.) Note that we have the adjacency $(u, v)$ in $G\left(N ; s_{1}, s_{2}\right)$ if and only if we have also the adjacency $(\phi(u), \phi(v))$ in $G\left(N ; s_{2}, s_{1}\right)$. We will call this isomorphism the direct isomorphism.

L-shaped tiles have been used as a metric tool to minimize the diameter of this kind of digraphs. Not only we can link an L-shaped tile to a given digraph $G$, but also we can recover the original digraph (or an isomorphical one) from its related tile. It is important to remark that the notation $L(l, h, w, y)$ not only completely describes the tile but also it gives the tiling which tessellates the plane. See $[2,4]$ for more details. The computation of the steps from the matrix $M$ is described in the following proposition (whose proof is contained in [4].)

Proposition 1 (Steps computation from the dimensions of the tile) Let $G$ be a double-loop with linked tile $L=L(l, h, w, y)$. Let $M$ be the matrix defining the tiling related to $L$, with $\operatorname{gcd}(l, h, w, y)=1$ and area $N=l h-w y$. Let $S(M)=\operatorname{diag}(1, N)$ be the Smith normal form of $M$, with related unimodular matrices $U$ and $V$ such that $S(M)=U M V$. Then the pair of steps $s_{1} \equiv U_{2,1}(\bmod N), s_{2} \equiv U_{2,2}(\bmod N)$ defines $G^{\prime}\left(N ; s_{1}, s_{2}\right)$ which is isomorphic to the original digraph $G$.

We will use this proposition later on. Two tiles are equivalent if they have the same area and the same number of nodes at any given distance from the node 0 . Two isomorphic digraphs have equivalent tiles, however two equivalent tiles can correspond to non isomorphical digraphs. Note that an isomorphic digraph to $G\left(N ; s_{1}, s_{2}\right)$ which is not the direct one must be of the form $G\left(N ; \zeta s_{1}, \zeta s_{2}\right)$ with $\zeta \in \mathbb{Z}_{N}^{\star}=\left\{n \in \mathbb{Z}_{N}: \operatorname{gcd}(n, N)=1\right\}$ the multiplicative group of unit elements in $\mathbb{Z}_{N}$, and their related tiles must be equivalent. Take for instance $N=5$ and the related tiles to $G(5 ; 1,2), G(5 ; 1,3)$ and $G(5 ; 2,3)$, respectively:

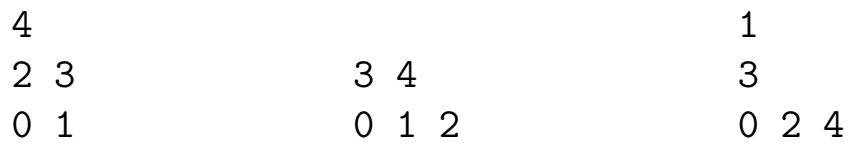

which are all equivalent ones, however $G(5 ; 2,3) \neq G(5 ; 1,2) \cong G(5 ; 1,3)$. Note that the digraph isomorphism $G(5 ; 1,2) \cong G(5 ; 3,1)$ is given by the unit $\xi=3$; also we have $G(5 ; 3,1) \cong G(5 ; 1,3)$ by the direct isomorphism, so we have $G(5 ; 1,2) \cong G(5 ; 1,3)$. There is no unit $\eta$ such that $(\eta 2, \eta 3)$ attains $(1,2)$ nor $(2,1)$, then $G(5 ; 2,3) \neq G(5 ; 1,2)$.

Although a great quantity of values of $N$ satisfy the identity $D(N)=D_{1}(N)$, there are infinite values of $N$ without this property. So we give the following definition.

Definition 2 (Nus integer) $N \in \mathbb{N}$ is a non-unit step (nus) integer if $D(N)<D_{1}(N)$. 


\begin{tabular}{|l||r|r|r|r|r|r|r|r|r|r|}
\hline$N$ & 450 & 924 & 930 & 1050 & 1764 & 2058 & 2415 & 2814 & 4224 & 4686 \\
\hline$D(N)$ & 35 & 51 & 51 & 55 & 71 & 77 & 84 & 91 & 111 & 117 \\
$s_{1}, s_{2}$ & 2,185 & 3,49 & 5,56 & 2,51 & 7,76 & 9,86 & 5,77 & 58,1437 & 1431,2827 & 75,3157 \\
\hline$D_{1}(N)$ & 36 & 52 & 52 & 56 & 72 & 78 & 85 & 92 & 112 & 118 \\
$s$ & 59 & 87 & 123 & 196 & 167 & 68 & 140 & 271 & 898 & 301 \\
\hline
\end{tabular}

Table 1: The first ten nus integers

The first published nus integer is $N=450$ (found in [5] by computer,) with $D(450)=$ $D(450 ; 2,185)=35$ and $D_{1}(450)=D(N ; 1,59)=36$. The first ten nus integers are given in the Table 1 and have been found by computer search. All of them correspond to tight digraphs unless $N=2814$ which is related to 1-tight optimal digraph. In this paper we propose a method to find infinite families of tight nus integers, that is integers $N$ with $D_{1}(N)>D(N)=\operatorname{lb}(N)$. Note that if $N$ is a nus integer then $D\left(N ; s_{1}, s_{2}\right)>D(N)$ if $\left\{s_{1}, s_{2}\right\} \cap \mathbb{Z}_{N}^{\star} \neq \emptyset$.

\section{Characterization of nus integers}

In order to find a characterization of nus integers, we will use the following results. Some of them are known yet in the bibliography.

Proposition 2 Let $L(l, h, w, y)$ be an L-shaped tile with area $N=l h-$ wy linked to the double-loop $G\left(N ; s_{1}, s_{2}\right)$. Then the steps $s_{1}, s_{2}$ satisfy the identity

$$
\left(\begin{array}{l}
s_{1} \\
s_{2}
\end{array}\right)=\left(\begin{array}{ll}
h & y \\
w & l
\end{array}\right)\left(\begin{array}{l}
\alpha \\
\beta
\end{array}\right),
$$

for some integral values $\alpha$ and $\beta$.

This Proposition was stated first in [5].

Lemma 1 Let $f(s, t)=a s+b t$ with $a, b \in \mathbb{N}$. If $g=f\left(s_{0}, t_{0}\right)>0$ is the least positive value of $f$ over all the integral values $s$ and $t$, then $\operatorname{gcd}(a, b)=g$.

The proof of this Lemma can be found in many basic texts on Number Theory.

Theorem 2 (Characterization of nus integers) $N \in \mathbb{N}$ is not a nus integer iff there is a tile $L(l, h, w, y)$ with area $N, l>y, h \geq w, d(L)=D(N)$ and $\operatorname{gcd}(l, w)=1$ or $\operatorname{gcd}(h, y)=1$.

\section{Proof:}

Suppose there is a such tile $L(l, h, w, y)$ with $l>y, h \geq w, d(L)=D(N)$ and $\operatorname{gcd}(l, w)=1$ (if $\operatorname{gcd}(l, w)>1$ and $\operatorname{gcd}(h, y)=1$, the proof is made by analogy.) Theorem 1 guarantees that $L$ realizes a double-loop digraph $G\left(N ; s_{1}, s_{2}\right)$ with $D\left(N ; s_{1}, s_{2}\right)=D(N)$. Now we must assure that $s_{1}=1$ or $s_{2}=1$. As $\operatorname{gcd}(l, w)=1$, there exist integers $s, t$ such that 
$s l-t w=1$. Let $M$ be the $2 \times 2$ integral matrix defining the tessellation from the tile $L(l, h, w, y)$, as it is described in the previous section. Then we have

$$
\left(\begin{array}{cc}
1 & 0 \\
s y-t h & 1
\end{array}\right) M\left(\begin{array}{cc}
s & w \\
t & l
\end{array}\right)=\operatorname{diag}(1, N)=S(M)
$$

and, from the Proposition 1, it follows that $D(N ; s y-t h(\bmod N), 1)=d(L)=D(N)$. Then we have $s_{1}=1$ and $L$ is $(1, s y-t h)$-implementable, so $D_{1}(N)=D(N)$ and $N$ is not a nus integer.

Now suppose $N$ a non nus integer. Then $D(N)=D_{1}(N)$. Let $L(l, h, w, y)$ with $l>y$ and $h \geq w$ be the related tile to the digraph $G(N ; s, 1)$ with $D(N ; s, 1)=D(N)$. By Proposition 2, we have

$$
\left(\begin{array}{l}
s \\
1
\end{array}\right)=\left(\begin{array}{ll}
h & y \\
w & l
\end{array}\right)\left(\begin{array}{c}
\alpha \\
\beta
\end{array}\right),
$$

for some $\alpha, \beta \in \mathbb{Z}$. Then we have $\alpha w+\beta l=1$. Now by Lemma 1 it follows that $\operatorname{gcd}(l, w)=1$

Theorem 2 characterizes nus integers in the negative sense, the following corollary characterizes them in the positive sense.

Corollary $1 N \in \mathbb{N}$ is a nus integer iff for any tile $L(l, h, w, y)$ with area $N$ and $d(L)=$ $D(N)$, we have

(a) $\operatorname{gcd}(l, w)>1$ and $\operatorname{gcd}(h, y)>1$,

(b) the identity $\operatorname{gcd}(l, h, w, y)=1$ and inequalities $l>y$ and $h \geq w$ are fulfilled for one of these tiles at least.

\section{Classification of tight nus integers}

The classification of tight nus integers will be done according to their related L-shaped tiles and it is based on the classification of tight tiles made in [4]. So we follow the notation used in this reference from now on. Let us denote by $x$ a non negative integer, then we define $I_{1}(x)=\left[3 x^{2}+1,3 x^{2}+2 x\right], I_{2}(x)=\left[3 x^{2}+2 x+1,3 x^{2}+4 x+1\right]$ and $I_{3}(x)=\left[3 x^{2}+4 x+2,3(x+1)^{2}\right]$. As $\mathbb{N}=\cup_{x=0}^{\infty}\left[3 x^{2}+1,3(x+1)^{2}\right]$, the closed intervals $I_{i}(x) i=1,2,3$ partition the set $\mathbb{N}$ for $x=0,1,2 \ldots$ Moreover $\operatorname{lb}(N)=3 x+i-2$ if $N \in I_{i}$ for $i=1,2,3$.

Following this parameterization, let us denote

$$
N_{i, j}(x, a, b)=3 x^{2}+(2 i+j-3) x+B_{i, j}(a, b)
$$

with $B_{i, j}(a, b)=a b-(a+b-i)(a+b+3-i-j)$, where $i$ stands for $N_{i, j}(x, a, b) \in I_{i}(x)$ and, as it is required in [4], $x \geq C_{i, j}(a, b)$ where

$$
C_{i, j}(a, b)=\left\lceil\frac{\alpha_{i}-B_{i, j}(a, b)}{j-1}\right\rceil, \quad \text { with } j \neq 1, \alpha_{1}=\alpha_{2}=1, \alpha_{3}=2 .
$$


Obvious restrictions must be added to the values $B_{i, j}(a, b)$ in order to assure $N_{i, j}(x, a, b) \in$ $I_{i}(x)$. According to the Table 2 in [4], there are nine different types of tight tiles: each type is denoted by $[i, j]$ for $i, j \in\{1,2,3\}$.

Theorem 3 (Classification of tight nus integers) If $N \in \mathbb{N}$ is a tight nus integer then all its related (tight) tiles $L(l, h, w, y)$ with $w \leq y$ are given by Table 2 with

$$
\begin{aligned}
\operatorname{gcd}(a+2 b-2 i, x-b+i) & >1, \\
\operatorname{gcd}(2 a+b+6-2 i-2 j, x-a+i+j-3) & >1,
\end{aligned}
$$

and at least one of these tiles has the parameters $x, a, b, i$ and $j \neq 1$ fulfilling the following conditions

$$
\operatorname{gcd}(a-b, 3 a-2 i, x+a+b-i, 3-j)=1, \quad l>y, \quad h \geq w .
$$

\section{Proof:}

If $N$ is a tight nus integer then it corresponds to the nodes of a tight DLN, so $D(N)=$ $\mathrm{lb}(N)$ and all its related (tight) tiles with $w \leq y$ are given by Table 2 in [4], that is Table 2 here. By Corollary 1 all these tiles must satisfy (3) and (4) which are equivalent to $\operatorname{gcd}(l(x, a), w(x, a, b))>1$ and $\operatorname{gcd}(h(x, b), y(x, a, b))>1$, respectively. Also by Corollary 1 , at least one of these tiles must satisfy (5).

According to Table 2 in [4], when $j=1$ (that is when $N$ is $N_{1,1}(x)=3 x^{2}+1, N_{2,1}(x)=$ $3 x^{2}+2 x+1$ or $\left.N_{3,1}(x)=3 x^{2}+4 x+2\right)$ we will see that at least one of its related tiles satisfies the Theorem 2 and so its related area, $N$, can not be a nus integer. Let us see this property in each of these three types of tiles:

- Type [1,1]: $N_{1,1}(x)=3 x^{2}+1$ have linked only one tile given by $l=h=2 x$, $w=x-1$ and $y=x+1$. The expression $N_{1,1}(x)$ corresponds to the nodes of a tight digraph if $\operatorname{gcd}(l, h, w, y)=1$ :

$$
\operatorname{gcd}(2 x, x-1, x+1)=\operatorname{gcd}(x-1, x+1)=\operatorname{gcd}(x-1,2)=1 \Leftrightarrow x \equiv 0 \quad(\bmod 2) .
$$

So we must restrict the values of $x$ to $x \equiv 0(\bmod 2)$. Then we have

$$
\operatorname{gcd}(l, w)=\operatorname{gcd}(2 x, x-1)=\operatorname{gcd}(2, x-1)=1 .
$$

- Type $[2,1]: N_{2,1}(x)=3 x^{2}+2 x+1$ has several related tiles, one of them is $l=h=$ $2 x+1 w=x$ and $y=x+2$. Then we have

$$
\operatorname{gcd}(l, w)=\operatorname{gcd}(2 x+1, x)=\operatorname{gcd}(1, x)=1 .
$$

- Type [3,1]: One of the tiles related to $N_{3,1}(x)=3 x^{2}+4 x+2$ is $L(2 x+1,2 x+$ $2, x, x+2)$. Also we have $\operatorname{gcd}(l, w)=\operatorname{gcd}(2 x+1, x)=1$. 


\begin{tabular}{|c|c|c|c|c|c|}
\hline$N_{i, j}(x, a, b)$ & $l(x, a)$ & $h(x, b)$ & $w(x, a, b)$ & $y(x, a, b)$ & $d(L)$ \\
\hline $\begin{array}{c}3 x^{2}+(2 i+j-3) x+B_{i, j}(a, b) \\
x \geq C_{i, j}(a, b)\end{array}$ & $2 x+a$ & $2 x+b$ & $x+a+b-i$ & $x+a+b+3-i-j$ & $3 x+i-2$ \\
\hline
\end{tabular}

Table 2: Data of a tile linked to a tight nus integer

So $N_{i, 1}(x)$ does not correspond to a nus integer for each $i=1,2,3$. So the value $j=1$ must be excluded from the possible options.

Table 2 must be understood with the usual restrictions mentioned above, as well as the additional restrictions on the integral pairs $(a, b)$ in order to have $0 \leq w(x, a, b) \leq l(x, a)$, $0 \leq y(x, a, b) \leq h(x, b), l(x, a)>0$ and $h(x, b)>0$. Theorem 3 characterizes tiles related to any tight nus integer. We will use this fact to describe an efficient method to find infinite families of tight nus integers containing any given value $N_{0}$ of such integers.

\section{A method to generate infinite families of tight nus integers}

Given a tight nus integer $N_{0}$, we can find all its related tiles with a time cost of $O\left(N_{0}^{1 / 2}\right)$ :

- $x_{0}$ and $i_{0}$ are found in constant time from $\operatorname{lb}\left(N_{0}\right)=3 x_{0}+i_{0}-2$,

- then the possible values of $j$ and $B_{j}=B_{i_{0}, j}(a, b)$ are found in constant time also,

- finally, for each value of $B_{j}$ all the possible values $(a, b)$ are found in time cost of $O\left(N_{0}^{1 / 2}\right)$ from the equation $a b-\left(a+b-i_{0}\right)\left(a+b+3-i_{0}-j\right)=B_{j}$, which represents an ellipse. According to the parameterization given in (2) we have $B_{j}=O\left(x_{0}\right)$ and $x_{0}=O\left(\sqrt{N_{0}}\right)$, and so $B_{j}=O\left(\sqrt{N_{0}}\right)$. Note that all possible integral points $(a, b)$ over this ellipse have their coordinates bounded by

$$
\left\lceil\frac{2\left(2 i_{0}+j-3\right)-\sqrt{\Delta}}{6}\right\rceil \leq a, b \leq\left\lfloor\frac{2\left(2 i_{0}+j-3\right)+\sqrt{\Delta}}{6}\right\rfloor
$$

with $\Delta=16\left(3-2 i_{0}-j\right)^{2}-48\left[B_{j}-i_{0}\left(i_{0}+j-3\right)\right]$. Then we can search all the possible integral values of $a$ (and $b$ also) in time cost $O(\sqrt{\Delta})$. Now from $\Delta=$ $O\left(B_{j}\right)=O\left(\sqrt{N_{0}}\right)$, we have that all possible integral points $(a, b)$ over the ellipse can be searched in time cost $O(\sqrt{\Delta}) O(\sqrt{\Delta})=O(\Delta)=O\left(\sqrt{N_{0}}\right)$.

In order to find an infinite family of nus integers containing the above given $N_{0}$, we must guarantee the following steps:

(a) Select the tiles satisfying (5) of Theorem 3.

(b) Find a subfamily, $N(\lambda)=N(x(\lambda))$, such that all the tiles found in (a) satisfy (3) and (4) for these values of $x(\lambda), \lambda \geq \lambda_{0}$, and $x\left(\lambda_{0}\right)=x_{0}$. 
(c) Finally, if the corresponding subfamily of related double-loop digraphs $G(\lambda)$ is required, compute the steps through the Proposition 1.

Note that if no subfamily is found in (b), by Theorem 3 there is no tight infinite family of nus integers containing the initial $N_{0}$.

Note that the condition $w \leq y$ given in Theorem 3 is not a restriction because if a given integer has a related tile $L(l, h, w, y)$ with associated DLN $G\left(N ; s_{1}, s_{2}\right)$, then it also has linked the tile $L(h, l, y, w)$ with associated DLN $G\left(N ; s_{2}, s_{1}\right)$ which is isomorphical to the other one. Note also that the exploration of tiles with $w \leq y$ in the method leads to the same conclusion as if all the tiles were searched, with a half time cost.

\subsection{Some application examples}

Now we can apply the method to the tight initial values of $N_{0}$ given in Table 1 , that is all values unless 2814 which is 1-tight optimal.

\begin{tabular}{|r|r|r|r|r|r|}
\hline$N_{0}$ & Type & $N(\lambda)$ & $s_{1}(\lambda)$ & $s_{2}(\lambda)$ & $D(N(\lambda))$ \\
\hline \hline 450 & {$[1,3]$} & $2700 \lambda^{2}+2220 \lambda+450$ & $90 \lambda+32$ & $90 \lambda+35$ & $90 \lambda+35$ \\
\hline 924 & {$[2,3]$} & $5292 \lambda^{2}+4452 \lambda+924$ & $3528 \lambda^{2}+2982 \lambda+622$ & $1764 \lambda^{2}+1512 \lambda+321$ & $126 \lambda+51$ \\
\hline 930 & {$[2,3]$} & $2700 \lambda^{2}+3180 \lambda+930$ & $90 \lambda+55$ & -3 & $90 \lambda+51$ \\
\hline 1050 & {$[3,2]$} & $1190700 \lambda^{2}+71190 \lambda+1050$ & 2 & $1890 \lambda+51$ & $1890 \lambda+55$ \\
\hline 1764 & {$[1,3]$} & $5292 \lambda^{2}+6132 \lambda+1764$ & $126 \lambda+80$ & 3 & $126 \lambda+71$ \\
\hline 2058 & {$[1,3]$} & $5292 \lambda^{2}+6636 \lambda+2058$ & -2 & $-2646 \lambda^{2}-3192 \lambda-959$ & $126 \lambda+77$ \\
\hline 2415 & {$[2,3]$} & $33075 \lambda^{2}+18060 \lambda+2415$ & 5 & $315 \lambda+77$ & $315 \lambda+84$ \\
\hline 4224 & {$[2,3]$} & $13068 \lambda^{2}+14916 \lambda+4224$ & $-4356 \lambda^{2}-4950 \lambda-1396$ & $-8712 \lambda^{2}-9900 \lambda-2793$ & $198 \lambda+111$ \\
\hline 4686 & {$[2,3]$} & $13068 \lambda^{2}+15708 \lambda+4686$ & 9 & $198 \lambda+130$ & $198 \lambda+117$ \\
\hline
\end{tabular}

Table 3: Infinite families of tight nus integers in Table 1 with $N_{0} \neq 2814$

Theorem 4 The nodes $N(\lambda), \lambda \geq 0$, of the infinite families of tight DLN appearing in Table 3 correspond to tight nus integers.

\section{Proof:}

We will develop the application of our method to the case $N_{0}=450$. We can proceed by analogy for the other cases, except for $N_{0}=1050$, so we will obviate their analysis.

For $N_{0}=450$, we have $\mathrm{lb}(450)=35$ that is $35=3 x-1$ for $x=12$, then $i=1$ (note that $450 \in I_{1}(12)$.) Now for $x=12$ it can be two possibilities:

$$
\begin{array}{ll}
j=2: & 3 x^{2}+x+B_{2}=450 \Rightarrow B_{2}=450-444=6, \\
j=3: & 3 x^{2}+2 x+B_{3}=450 \Rightarrow B_{3}=450-456=-6 .
\end{array}
$$

Then we must search for the solutions of the equations $B_{1,2}(a, b)=6$ and $B_{1,3}(a, b)=-6$. The former has no solutions and the latter has six, with the associated tight tiles given by 


\begin{tabular}{|rllll|}
\hline$(a, b)$ & $l(x)$ & $h(x)$ & $w(x)$ & $y(x)$ \\
\hline$(1,3)$ & $2 x+1$ & $2 x+3$ & $x+3$ & $x+3$ \\
$(3,1)$ & $2 x+3$ & $2 x+1$ & $x+3$ & $x+3$ \\
$(-2,3)$ & $2 x-2$ & $2 x+3$ & $x$ & $x$ \\
$(3,-2)$ & $2 x+3$ & $2 x-2$ & $x$ & $x$ \\
$(-2,1)$ & $2 x-2$ & $2 x+1$ & $x-2$ & $x-2$ \\
$(1,-2)$ & $2 x+1$ & $2 x-2$ & $x-2$ & $x-2$ \\
\hline
\end{tabular}

for $x \geq C_{1,3}(a, b)=\left\lceil\frac{1-B_{1,3}(a, b)}{2}\right\rceil=4$. Now we must compute the gcd of the dimensions of the tiles:

$(1,3): \quad \operatorname{gcd}(2 x+1,2 x+3, x+3)=\operatorname{gcd}(2 x+1, x, x+3)=\operatorname{gcd}(1, x, x+3)=1 \quad \forall x \geq 4$, $(-2,3): \quad \operatorname{gcd}(2 x-2,2 x+3, x)=\operatorname{gcd}(-2,3, x)=1 \quad \forall x \geq 4$,

$(-2,1): \quad \operatorname{gcd}(2 x-2,2 x+1, x-2)=\operatorname{gcd}(x, 2 x+1, x-2)=\operatorname{gcd}(x, 1,-2)=1 \quad \forall x \geq 4$.

Inequalities $l(x)>y(x)$ and $h(x) \geq w(x)$ are fulfilled for $x \geq 4$. Then all the found tiles have passed the step (a) of the method.

Now we must certify the conditions given in the step (b) for all the tiles. The first one gives us the conditions

$\operatorname{gcd}(l, w)=\operatorname{gcd}(2 x+1, x+3)=\operatorname{gcd}(x-2, x+3)=\operatorname{gcd}(x-2,5)=5$ if $x \equiv 2(\bmod 5)$, $\operatorname{gcd}(h, y)=\operatorname{gcd}(2 x+3, x+3)=\operatorname{gcd}(x, x+3)=\operatorname{gcd}(x, 3)=3$ if $x \equiv 0 \quad(\bmod 3)$.

Proceeding in the same way, the other tiles add the condition $x \equiv 0(\bmod 2)$. Now we must solve the system of congruences

$$
\left\{\begin{array}{l}
x \equiv 0(\bmod 2) \\
x \equiv 0(\bmod 3) \\
x \equiv 2(\bmod 5)
\end{array}\right.
$$

which, by the Chinese reminder theorem, has the solution $x \equiv 12(\bmod 30)$. So we have $x(\lambda)=30 \lambda+12$ for $\lambda \geq 0$.

Let us now compute the steps of the corresponding double-loop digraph. We will use the Proposition 1 applied to any of the six found tiles. Let us take the first one and let us consider the matrix $M=\left(\begin{array}{rr}2 x+1 & -x+2 \\ -x+2 & 2 x-2\end{array}\right)$. Now we must compute a required unimodular matrix $U$ as it is described in the Proposition 1. Since

$$
S(M)=\operatorname{diag}\left(1,3 x^{2}+2 x-6\right)=\left(\begin{array}{rr}
x-1 & x \\
3 x-4 & 3 x-1
\end{array}\right) M\left(\begin{array}{rr}
1 & -x^{2}-x+2 \\
-1 & x^{2}+x-1
\end{array}\right),
$$

we have $s_{1}(x)=3 x-4(\bmod N)$ and $s_{2}(x)=3 x-1(\bmod N)$. Finally, from the substitution $x=x(\lambda)$, the stated expressions of $N(\lambda), s_{1}(\lambda), s_{2}(\lambda)$ and $D(N(\lambda))$ in Table 3 , for $N_{0}=450$, are derived. These values are valid for $\lambda \geq 0$ (note that $x(0)=12 \geq 4$.) The 
other two types $[1,1]$ and $[1,2]$ can not contain any tight tile with area $N(x)=3 x^{2}+2 x-6$ for $x \geq 4$ because $B_{i, j}(a, b) \leq\left\lfloor\frac{(2 i+j-3)^{2}}{3}\right\rfloor+i(3-i-j) \forall(a, b) \in \mathbb{Z}^{2}$.

When $N_{0}=1050$, it belongs to the family $N_{3,2}(x, a, b)=3 x^{2}+5 x-12$ for several pairs $(a, b)$. By analogy with the above cases we can find the subfamily (when $x=210 \lambda+18$ )

$$
\begin{aligned}
N(\lambda) & =132300 \lambda^{2}+23730 \lambda+1050 \\
s_{1}(\lambda) & =2 \\
s_{2}(\lambda) & =630 \lambda+51 \\
D(N(\lambda)) & =630 \lambda+55
\end{aligned}
$$

with no $(1, s)$-implementable tight tiles of type [3,2]. However it could be possible to represent many values of $N(\lambda)$ by $(1, s)$-implementable tight tiles of type [3,3]. Now we will restrict this subfamily in order that such a representation does not occur.

Let us see that type $[3,3]$ does not represent any value of $N(\lambda)$ when $\lambda \equiv 0(\bmod 3)$. A necessary condition for type $[3,3]$ to represent the area $3 x^{2}+5 x-12$, for a certain $x$, is $B_{3,3}(a, b)=-x-12$. Let us see that this equality is not possible for $x=210 \lambda+18$ and $\lambda=3 \lambda^{\prime}$. We must prove the impossibility of

$$
a b-(a+b-3)^{2}=-630 \lambda^{\prime}-30,
$$

for integral values of $a, b$ and $\lambda^{\prime} \geq 0$. Noting that $-630 \lambda^{\prime}-30 \equiv 0(\bmod 3)$, we have

$$
\begin{aligned}
& a \equiv 0(\bmod 3) \Leftrightarrow b \equiv 0(\bmod 3), \\
& a \equiv 1(\bmod 3) \Leftrightarrow b \equiv 1(\bmod 3), \\
& a \equiv 2(\bmod 3) \Leftrightarrow b \equiv 2(\bmod 3) .
\end{aligned}
$$

For $a \equiv b \equiv 0(\bmod 3)$, we substitute $a=3 a^{\prime}, b=3 b^{\prime}$ and expression $(6)$ derives in

$$
9 a^{\prime} b^{\prime}-9\left(a^{\prime}+b^{\prime}-1\right)=-9 \times 70 \lambda^{\prime}-3 \times 10,
$$

which is a contradiction because 9 does not divide the value $3 \times 10$.

If $a \equiv b \equiv 1(\bmod 3)$, by the substitution $a=3 a^{\prime}+1$ and $b=3 b^{\prime}+1$ the expression $(6)$ becomes

$$
9 a^{\prime} b^{\prime}+9\left(a^{\prime}+b^{\prime}\right)-9\left(a^{\prime}+b^{\prime}\right)^{2}=-9 \times 70 \lambda^{\prime}-3 \times 10,
$$

a contradiction like in the above case.

Finally, for $a \equiv b \equiv 2(\bmod 3)$, the substitution $a=3 a^{\prime}+2$ and $b=3 b^{\prime}+2$ turns the expression (6) to be

$$
9 a^{\prime} b^{\prime}-9\left(a^{\prime}+b^{\prime}\right)=-9 \times 70 \lambda^{\prime}-3 \times 11,
$$

which is also a contradiction because 11 is a prime number, not divisible by 3 . Then the subfamily we have been looking for is given by the substitution $\lambda=3 \lambda^{\prime}$. 


\section{$5 \quad$ Remarks}

One of the first family of tight nus integers was published in [4] as an application example of the Tight Tiles Table given in that work. That family corresponds to the one given in Table 1 with $N_{0}=450$. Although $N(\lambda)$ is a correct value, an erratum appears in the expression of the steps in [4]. A possible correct pair of steps is given here in Table 1. Note that, for $\lambda=0$, we obtain the DLN $G(450 ; 32,35)$ which isomorphic to $G(450 ; 2,185)$ appearing in [4] (this isomorphism is given by the unit $211 \in \mathbb{Z}_{450}^{\star}: 211 \times 32 \equiv 2(\bmod 450)$ and $211 \times 35 \equiv 185(\bmod 450)$.) This erratum is remarked without any loss of validity on the generic results stated there.

There are some flaws in Theorem 3 in [6]. The diameter of $G(10688 ; 3,57)$ is 579 , not 59 as it is stated there. Moreover the stated digraph is isomorphic to $G(10688 ; 1,19)$ by the unit $3563 \in \mathbb{Z}_{10688}^{\star}$. However all these flaws seem to be only several errata because the expressions of the nodes, second step and diameter, for $t=84 e+59$, would be

$$
\begin{aligned}
N(e) & =3 t^{2}+4 t-11=21168 e^{2}+30072 e+10668 \\
s_{2}(e) & =3 t-2=252 e+175 \\
D\left(N(e) ; 3, s_{2}(e)\right) & =3 t=252 e+177
\end{aligned}
$$

which are different from those given there.

All first ten nus integers listed here are such that the identity $D_{1}(N)=D(N)+1$ holds. However there exist others with $D_{1}(N)-D(N) \geq 2$. The first nus integer with $D_{1}(N)=D(N)+2$ is $N=11382$, found by computer search, with $D(11382)=$ $D(11382 ; 1634,3269)=183$ and $D_{1}(11382)=D(11382 ; 1,2238)=185$. As $\operatorname{lb}(11383)=$ 183 , this is a 2-tight nus integer.

Acknowledgment. Authors thank Prof. Chiuyuan Chen for her accurate comments and detailed suggestions.

\section{References}

[1] F. Aguiló and M.A. Fiol, An efficient algorithm to find optimal double loop networks, Discrete Math. 138 (1995) 15-29.

[2] J.-C. Bermond, F. Comellas and D.F. Hsu, Distributed loop computer networks: A survey, J. Parallel Distrib. Comput. 24 (1995) 2-10.

[3] C. Chen and F.K. Hwang, The minimum distance diagram of double-loop networks, IEEE Trans. on Computers Vol. 49 (2000) 977-979.

[4] P. Esqué, F. Aguiló and M.A. Fiol, Double commutative-step digraphs with minimum diameters, Discrete Math. 114 (1993) 147-157. 
[5] M.A. Fiol, J.L.A. Yebra, I. Alegre and M. Valero, A discrete optimization problem in local networks and data alignment, IEEE Trans. Comput. C-36 (1987) 702-713.

[6] Xu J., Designing of optimal double loop networks, Science in China, Series E, vol. E42 num. 5 (1999) 462-469.

[7] C.K. Wong and D. Coppersmith, A combinatorial problem related to multimode memory organizations, J. Ass. Comput. Match. 21 (1974) 392-402. 